MODELING, IDENTIFICATION AND CONTROL, 1983, vOL. 4, NO. 1, 1-23

doi:10.4173/mic.1983.1.1

\title{
GPS performance in marine applications
}

\author{
JOHNNY BARDAL† and OLE ØRPEN†
}

Keywords: Satellite navigation, Kalman filters, digital simulation, surface navigation.

This paper presents simulations of Global Positioning System (GPS) receiver performance for marine applications. An overview of the GPS system along with a detailed description of the GPS receiver Kalman filter implementation is given. Also presented is a description of ship behaviour in a seaway. The end of the paper presents a few selected simulation results giving the performance of a GPS receiver for marine applications.

\section{Introduction}

The Navstar GPS is a space-based radio navigation and time-transfer system that is being developed by the US Department of Defense to provide highly precise position, velocity and time information to users anywhere and at any time. The GPS Program is a multi-service effort and is managed by the GPS Joint Program Office (JPO) at US Air Force Space Division in Los Angeles, California. The United States encouraged NATO participation in the development and deployment of the GPS. In response, ten NATO nations-including Norway-signed a Memorandum of Understanding (MOU) in June 1978 for participation in the development of GPS. The objective of the MOU is to establish a flow of information among the participating nations in all GPS activities.

At the March 1981 meeting the NATO Navstar GPS Steering Committee approved partial funding for a one year study on the integration of GPS and inertial navigation systems for marine applications. The study was proposed by the Norwegian Defence Research Establishment.

The study was prepared for the NATO Deputy Program Manager at the JPO in Los Angeles. This program office was the managing agency for the study on behalf of the NATO Navstar Steering Committee. This paper presents selected results from the final study report (Bardal and Ørpen 1982) showing different GPS receivers operating alone and integrated with an attitude reference system. In the following an introduction to the Navstar GPS satellite navigation system is given. A brief description of the models used in the simulations of the environment and a description of the filter implementation will also be given. Finally, some simulation results will be presented as an indication of the GPS receiver performance in different situations. The examples include lever arm effects in a seaway and satellite outage caused by mountain shadowing.

\section{GPS system overview}

A simplified overview of the GPS system concept is given in Fig. 1. Full system capability will be achieved in 1988 when 18 satellites will be operational. At this time

Received 19 October 1982.

† Norwegian Defence Research Establishment, P.O. Box 25, N-2007 Kjeller, Norway. 


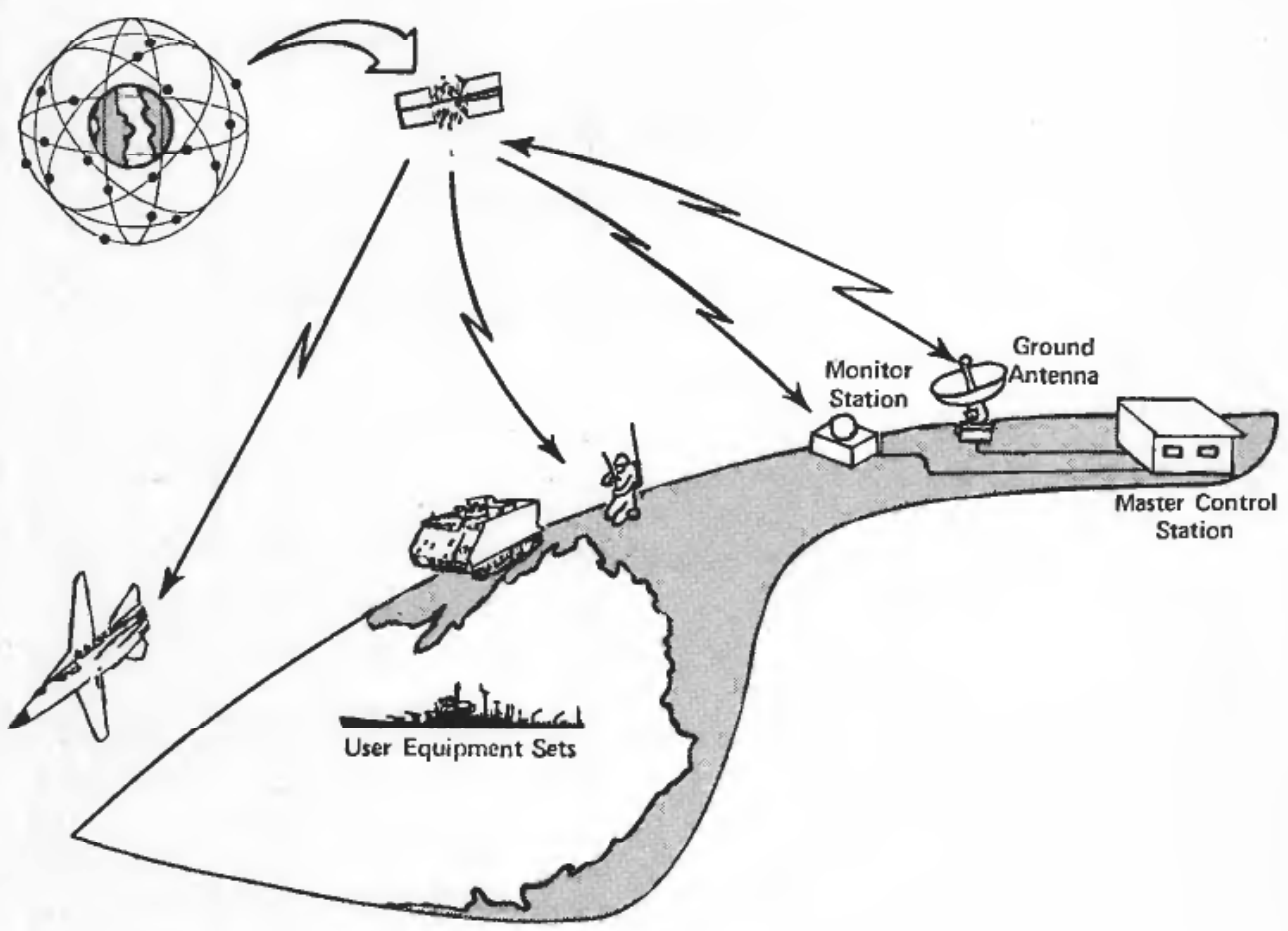

Figure 1. The GPS system.

the GPS system will be capable of providing the user with instantaneous navigation and time data with $10 \mathrm{~m}(\mathrm{CEP})$ position, $0.1 \mathrm{~m} / \mathrm{s}(\mathrm{RMS})$ velocity and $0.1 \mu \mathrm{s}(1 \sigma)$ time accuracy.

Each satellite (often termed Space Vehicle or SV) transmits two signals on the frequencies $1575 \mathrm{MHz}\left(L_{1}\right)$ and $1227 \mathrm{MHz}\left(L_{2}\right)$. Using two frequencies makes it possible to compensate for the signal delay through the ionosphere.

The $L_{1}$ signal will carry a $P$ (Precise or Protected) code and a $C / A$ (Clear or Coarse/Acquisition) code modulated onto the carrier. The $L_{2}$ signal will carry either a $P$ or a $C / A$ code. The $P$ and $C / A$ codes are pseudo-random bit sequences (i.e. the bit value is made to look like it changes at random) which are biphase modulated onto the carrier (each time the bit value changes the carrier phase is reversed $180^{\circ}$ ). The $P$ code will be made available only to authorized users while the $C / A$ code will be made available to the public. Also, the $C / A$ code is used to acquire the $P$ code. The bit rate of the codes are $10.23 \mathrm{MHz}(P)$ and $1.023 \mathrm{MHz}(C / A)$. The accuracies specified above are those obtained by $P$ code navigation. By $C / A$ code navigation an accuracy of $200 \mathrm{~m}$ (CEP) will be made available to the public even though the inherent capability of the $C / A$ code is much better. Superimposed on the $P$ and $C / A$ codes there will be a navigation and system data message. This message will include satellite cphemeris, atmospheric propagation correction data and satellite clock bias information.

In order to navigate a user needs to track four satellites. By measuring the propagation time needed for the signals to reach the user, the distance to the four satellites may be inferred. This necessitates a close synchronization between the satellite and user clocks. This is the reason why four satellites are needed; in addition 
to calculating a three dimensional position, the bias in the user clock must be determined. In a marine application the fact that a vessel is on the ocean surface may be used, and three satellites will be sufficient to determine the horizontal position and the receiver clock bias.

There will be two main categories of GPS receivers; parallel and sequential receivers. A parallel receiver tracks four satellites simultaneously on separate channels, while a sequential receiver tracks the four satellites in sequence on one channel. This generally means that a parallel receiver gets a new position fix four times as often as a sequential receiver, with $I$ and 4 seconds intervals respectively for the simulation results presented. Parallel receivers will generally be used on high dynamic vehicles while sequential receivers will be used on medium and low dynamic vehicles.

The receiver measures the distance to the satellites by tracking the $P$ or $C / A$ code. This distance is termed pseudorange to imply that it contains the unknown user clock bias. Also the receiver measures the velocity along the line of sight between the user and the satellite. This is done by tracking the carrier signal and measuring the doppler frequency shift on this signal. By compensating for the known satellite velocity the receiver velocity may be calculated. The doppler measurement is usually implemented by integrating the doppler over a short time period (e.g. 0.5 seconds). The measure resulting from this is called pseudodeltarange or just deltarange. The term pseudo here implies that the measure also contains an unknown user clock frequency error. The pseudorange and the deltarange are used in the navigation software to update the position and the velocity of the receiver. Table 1 presents error summaries for the pseudorange ( $P$ code) and deltarange. The error budget presented is consistent with the one presented in the GPS system specification. Subsequent to the simulations presented below were performed, updated expected GPS errors have been provided. The new values include $3 \mathrm{~m}$ (old $5.3 \mathrm{~m}$ ) for the satellite clock and $4.3 \mathrm{~m}(2.8 \mathrm{~m})$ for ephemeris giving a total ranging error of $6.5 \mathrm{~m}(7 \mathrm{~m})$. These changes will only have a minor influence on the results presented.

\begin{tabular}{|c|c|c|c|c|}
\hline \multirow[t]{2}{*}{ Error contributor } & \multicolumn{2}{|c|}{ Pseudorange } & Deltarange & \multirow[t]{2}{*}{ Statistics } \\
\hline & $1 \sigma$ & $\begin{array}{l}\text { time } \\
\text { const }\end{array}$ & $\begin{array}{l}\text { time } \\
\text { const }\end{array}$ & \\
\hline \multirow[t]{2}{*}{ Satellite clock } & $5 \cdot 3 \mathrm{~m}$ & & & $\begin{array}{l}\text { Random walk } \\
\text { + random ramp }\end{array}$ \\
\hline & & & $6.0 \cdot 10^{-5} \mathrm{~m} / \mathrm{s}$ & Random constant \\
\hline Satellite ephemeris & $2 \cdot 8 \mathrm{~m}$ & & & Random constant \\
\hline Ionospheric error & $2 \cdot 3 \mathrm{~m}$ & $60 s$ & & Exp. correlated \\
\hline Tropospheric error & $2 \cdot 0 \mathrm{~m}$ & & & Random constant \\
\hline $\begin{array}{l}\text { Propagation } \\
\text { gradient }\end{array}$ & & & $\begin{array}{l}1 \cdot 1 \cdot 10^{-2} \mathrm{~m} / \mathrm{s} 60 \mathrm{~s} \\
\quad \text { (evaluated at } 5^{\circ} \\
\text { elevation) }\end{array}$ & Exp. correlated \\
\hline Multipath & $1.2 \mathrm{~m}$ & & & Uncorrelated \\
\hline Measurement error & $1.5 \mathrm{~m}$ & & $0.02 \mathrm{~m}$ & Uncorrelated \\
\hline
\end{tabular}

Table 1. Pseudorange and deltarange error summary. 
The magnitude of the ranging errors combined with the geometry of the selected satellites will determine the magnitude of the user position errors in the GPS navigation fix. The effect of the geometry is expressed by the GDOP (Geometric Dilution of Precision) parameters. These parameters include:

$$
\begin{aligned}
& \text { PDOP-Position Dilution of Precision } \\
& \text { HDOP-Horizontal Dilution of Precision } \\
& \text { VDOP-Vertical Dilution of Precision } \\
& \text { TDOP-Time Dilution of Precision }
\end{aligned}
$$

If the user to satellite ranging error is named RE one can set up the following definitions:

PDOP * RE-radial position error in 3 dimensions

HDOP $*$ RE-radial position error in the horizontal plane

$\mathrm{VDOP} * \mathrm{RE}-$ vertical position error

TDOP * RE-error in the user clock

A short description of these parameters is given in Appendix A. Comments on the model of the GPS system are given below.

For the SV clock a cesium standard has been assumed with a relative frequency stability of $2 \cdot 10^{-13}$. The delay of the Navstar signals in the ionosphere is inversely proportional to the square of the frequency. By transmitting two frequencies it is therefore possible for the receiver to compensate for the ionospheric delay. There will, however, be a residual error after the two frequency compensation. This error is mostly due to measurement noise. It was assumed that the ionospheric correction is lowpass filtered to reduce this noise. The time constant in such a filter should not be too long in order to accommodate changes in the ionosphere. A time constant of 1 min was assumed.

Propagation gradients through the ionosphere and the troposphere will influence the deltarange measurements of the GPS system. The error model given below was assumed to take both ionospheric and tropospheric gradients into account, although the ionospheric gradient probably will be the bigger of the two.

$$
\delta=\frac{K_{g}}{\sin (E)}
$$

$\delta$ - delta range error

$E$-elevation angle towards the satellite

$K_{g}$-propagation gradient proportionality factor

$K_{g}$ was modeled as an exponentially correlated noise with standard deviation $\sigma_{K_{g}}=0 \cdot 5 \cdot 10^{-3} \mathrm{~m} / \mathrm{s}$.

The GPS system has an inherent capability to reject multipath signals that have a delay more than 1.5 of a code chip of the direct signal. If the delay is smaller an error in pseudorange will occur. The multipath tends to be very sporadic in nature and is dependent on vehicle attitude, antenna gain etc. The simulation program includes multipath related errors as an uncorrelated error with zero mean. The size of the error was $1.2 \mathrm{~m}$ ( $P$ code). 
A GPS receiver consists of two kinds of tracking loops. One of them tracks the pseudorandom code that is modulated on the carrier and the other tracks the carrier. The code tracking loop provides the pseudorange to the SV while the carrier tracking loop provides the deltarange. These tracking loops tend to introduce a random error on the measurement. The size of these errors are dependent on receiver design. The pseudorange tracking error allocated is $1.5 \mathrm{~m}(1 \sigma)$. This error applies to $P$ code tracking, and it was assumed to be random. 1/10 of a carrier cycle is allocated to the deltarange tracking error. This corresponds to approximately $2 \mathrm{~cm}$ on the $L_{1}$ frequency $(1575 \mathrm{MHz})$. The deltarange error was in the simulation assumed to be a random error of $2 \mathrm{~cm}(1 \sigma)$.

The user clock was modeled assuming a crystal oscillator. The model contains an ageing term, a correlated noise term and an acceleration dependent term in addition to white frequency noise. The most important parameters of this model were the relative frequency error of $10^{-11}$ and the $g$-sensitivity of $10^{-9} / g$.

\section{Ship motions}

The motions of a ship are usually considered to occur in the six degrees of freedom known as surge, sway, heave, roll, pitch and yaw. The motions are coupled in a manner that depends on the wave direction and the ship speed. It is generally accepted that the responses of a ship to irregular waves can be considered as a summation of the responses to regular waves of all frequencies. The problem of predicting ship motions in a seaway can then be reduced to the prediction of the ship motions in regular sinusoidal waves, and then combine these regular wave results and a description of the seaway to generate the statistical responses in irregular waves. The best way to define a seaway is by means of a wave energy spectrum. The sea spectrum used in this study is that adopted by the International Towing Tank Conference (ITTC). The spectrum is (Price et al. 1974):

$$
\Phi(\omega)=\frac{A}{\omega^{5}} \exp \left(-B / \omega^{4}\right)
$$

where

$$
\begin{aligned}
& \Phi \text {-energy density }\left[\mathrm{m}^{2} \mathrm{~s}\right] \\
& \omega-\text { angular frequency }[\mathrm{rad} / \mathrm{s}] \\
& A=8 \cdot 1 \cdot 10^{-3} \mathrm{~g}^{2} \\
& B=\frac{3 \cdot 11}{h_{1 / 3}^{2}} \\
& g \text {--acceleration of gravity }\left[\mathrm{m} / \mathrm{s}^{2}\right] \\
& h_{1 / 3} \text { - significant wave height [m] }
\end{aligned}
$$

The sea condition is designated by the significant wave height, which is the average height of the one-third highest waves in the seaway. The spectrum is illustrated in Fig. 2.

The wave spectrum is divided into sinusoidal components as shown in Fig. 3. The frequency of each component sinusoidal wave is the centre frequency of its histogram. The amplitude of each component is given by the formula below:

$$
A_{i}=\left(2 \Phi\left(\omega_{i}\right) \Delta \omega_{i}\right)^{1 / 2}
$$




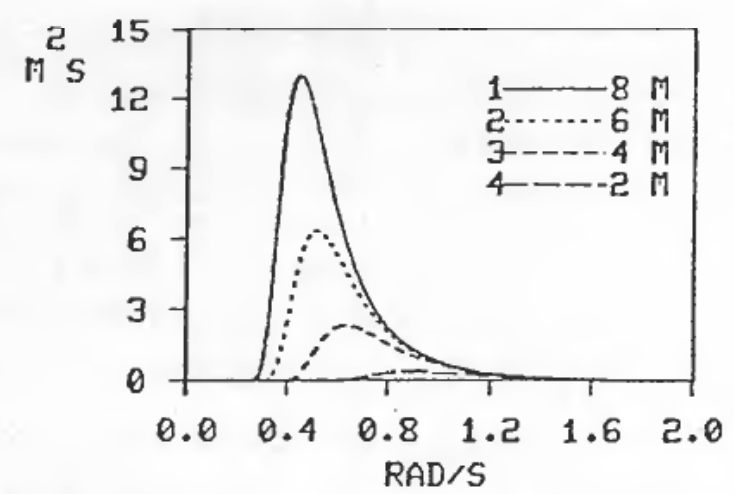

Figure 2. The standard wave spectrum $\Phi(\omega)$ of the International Towing Tank Conference for the various values of the significant wave height.

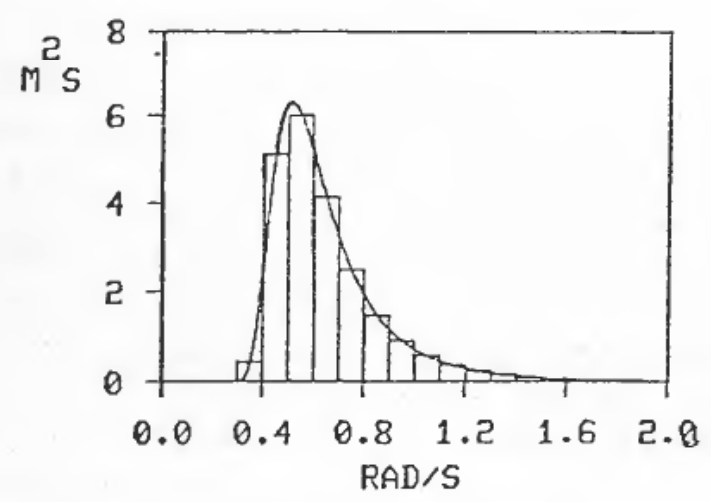

Figure 3. The wave spectrum $\Phi(\omega)$ divided into sinusoidal components.

where

$A_{i}$-amplitude of the $i$ component sinusoidal wave

$\Phi\left(\omega_{i}\right)$ - energy density for $\omega_{i}$

$\omega_{i}$-centre frequency of histogram $i$

$\Delta \omega_{i}$-frequency interval covered by histogram $i$

It is generally assumed that all phase angles are equally probable. That is to say that the wave phase for each component sinusoidal wave is uniformly distributed between 0 and $2 \pi$ and there is no phase correlation between wave components. For deep water waves we have the following relation between wavelength $\lambda[\mathrm{m}]$ and wave frequency $\omega[\mathrm{rad} / \mathrm{s}]$ (Price et al. 1974):

$$
\omega=\left(\frac{2 \pi g}{\lambda}\right)^{1 / 2}
$$

Response data for a frigate was used. The ship motions in six degrees of freedom as a function of wavelength were generated. The motions were represented by amplitude values and phase lags with respect to the time the wave hits midship. 
The ship response has to be expressed in terms of wave encounter frequencies. Therefore, the wave frequencies have to be doppler shifted for the ship's speed component in the direction of the wave. For deep water waves the following relation for the wave encounter frequency applies (Price et al. 1974):

$$
\omega_{e}=\omega-\omega^{2} v \cos (\theta) / g
$$

where

$\omega_{e}$-wave encounter frequency (doppler shifted angular frequency) [rad/s]

$\omega-$ wave angular frequency seen by a stationary observer [rad/s]

$v$-ship speed $[\mathrm{m} / \mathrm{s}]$

$\theta$ - the ship heading with respect to the waves

Compared to $\omega, \omega_{e}$ is lower for following seas, the same for beam seas and higher for head seas.

Lever arm effects occur when the antenna location and the ship's centre of gravity do not coincide. In the simulation the antenna was assumed to be positioned directly above the centre of mass of the frigate. The height above the centre of mass used was $18 \mathrm{~m}$.

\section{Kalman filter development}

This section contains the description of the GPS receiver Kalman filter used in the study. First a general description of the Kalman filter is presented and the notation used defined. Next, a few considerations on the implementation of the filter in the simulation program are presented. Then the filter is described in more detail.

\subsection{General dynamic model and Kalman filter description}

The dynamic model and the Kalman filter will be given on a general form below. The notation used has been adopted from Gelb (1974).

The continuous model of dynamic equations is:

$$
\begin{aligned}
\dot{x} & =F x+G w \\
z & =H x+v \\
E\left(x x^{T}\right) & =P \\
E\left([G w][G w]^{T}\right) & =G Q G^{T} \delta(t-\tau) \\
E\left(v v^{T}\right) & =R \\
E(v)=E(w) & =0
\end{aligned}
$$

All the variables may be functions of time and are described as:

$$
\begin{aligned}
& x \text {-error state vector } \\
& \boldsymbol{w} \text { - } \text { - plant noise vector, white gaussian noise } \\
& z \text {-measurement vector } \\
& \boldsymbol{y} \text {-measurement noise vector, white gaussian noise }
\end{aligned}
$$




$$
\begin{aligned}
& F \text {-system matrix } \\
& G \text { - plant noise matrix } \\
& H \text {-measurement matrix } \\
& P \text {--error covariance matrix of state vector } \\
& Q \text { - spectral density matrix of plant noise } \\
& R \text { - measurement noise variance matrix } \\
& E \text { - the expectation operator } \\
& \delta(t-\tau) \text { - Dirac delta function }
\end{aligned}
$$

The dynamic models used in the simulation will be presented in a continuous form. The simulation software includes algorithms for discretizing the continuous models. The discrete form of the dynamic models is:

$$
\begin{aligned}
x_{k} & =\Phi_{k-1} x_{k-1}+\Gamma_{k-1} w_{k-1} \\
z_{k} & =H x_{k}+v_{k} \\
E\left(x_{k} x_{k}^{T}\right) & =P_{k} \\
E\left(\left[\Gamma_{k} w_{k}\right]\left[\Gamma_{k} w_{k}\right]^{T}\right) & =\Gamma_{k} Q_{k} \Gamma_{k}^{T} \\
E\left(v_{k} v_{k}^{T}\right) & =R_{k}
\end{aligned}
$$

where

$$
\begin{aligned}
& k \text {-time step index } \\
& \Phi \text {-transition matrix } \\
& Q_{k} \text {-discrete plant noise covariance matrix }
\end{aligned}
$$

and the other matrices and vectors are discrete versions of the continuous ones.

The conventional Kalman filter is given below (Gelb 1974).

Time update:

$$
\left.\begin{array}{l}
\hat{\boldsymbol{x}}_{k}(-)=\Phi_{k-1} \hat{\boldsymbol{x}}_{k-1}(+) \\
P_{k}(-)=\Phi_{k-1} P_{k-1}(+) \Phi_{k-1}{ }^{T}+\Gamma_{k-1} Q_{k-1} \Gamma_{k-1}{ }^{T}
\end{array}\right\}
$$

Measurement update:

$$
\left.\begin{array}{rl}
\hat{x}_{k}(+) & =\hat{\boldsymbol{x}}_{k}(-)+K_{k}\left(z_{k}-H_{k} \hat{र}_{k}(-)\right) \\
P_{k}(+) & =\left(I-K_{k} H_{k}\right) P_{k}(-) \\
K_{k} & =P_{k}(-) H_{k}{ }^{T}\left[H_{k} P_{k}(-) H_{k}{ }^{T}+R_{k}\right]^{-1}
\end{array}\right\}
$$


where

$\hat{\boldsymbol{x}}_{\boldsymbol{k}}(-), \hat{\boldsymbol{x}}_{\boldsymbol{k}}(+)$-prior and updated estimates of the error state vector

$P_{k}(-), P_{k}(+)$-prior and updated estimates of the error state covariance

$K_{k}-$ Kalman gain

The Kalman filter algorithm implemented is a mathematically equivalent, but a numerically more stable algorithm than the one presented above. It is called the $U-D$ factorization algorithm and it is described in Biermann (1977). The idea of this algorithm is to factorize the covariance matrix in the following manner:

$$
P=U D U^{T}
$$

where $U$ is an upper triangular matrix and $D$ is a diagonal matrix. Instead of propagating $P$, the $U$ and $D$ factors are now propagated. Detailed algorithms for the propagation are given in Biermann (1977).

\subsection{Filter implementation considerations}

This subsection will give a rationale for the number of error states selected in the filter. The error state vector selected in the Kalman filter has more error states than a practical implementation of such a filter would have. In an 'optimal' filter implementation all the error sources of the environment should be modeled. However, due to computer throughput and memory limitations, generally only those errors that can be estimated are modeled in the filter. Then the plant noise of the remaining error states and the measurement noise are adjusted in order to get a filter performance close to the 'optimal' filter. This is called filter tuning.

The SV range bias error states described in the next sections, are examples of such additional error states that would not be implemented in a practical system. The reasons for using additional states may be summed up as:

1. One avoids extensive non-optimal tuning as the extended filter is in closer agreement with the actual errors. In a practical implementation this tuning will also be adaptive to accommodate changes in the external conditions.

2. One gets Kalman filter indicated covariances which are in closer agreement with the actual covariances. This reduces the need to perform time consuming Monte Carlo simulations, it will only be necessary to do that in selected instances to verify the performance.

3. The extended filter performance sets the performance limit of a well tuned reduced state filter.

Additionally, it was not the aim of this study to develop a filter that can be fitted directly into a piece of user equipment. Also, these additional states do not add excessively to the simulation time required.

\subsection{GPS Kalman filter dynamic error model}

The GPS receiver Kalman filter has 16 error states. The reference system used is the local level north, east, down system. The error states are defined as the difference 
between computed values as given by the GPS receiver, and true values. The dynamic error model used in the GPS receiver Kalman filter is given below.

$$
\begin{aligned}
\delta r_{N} & =\delta v_{N} \\
\delta r_{E} & =\delta v_{E} \\
\delta r_{D} & =\delta v_{D} \\
\delta v_{N} & =\delta a_{N} \\
\delta v_{E} & =\delta a_{E} \\
\delta v_{D} & =\delta a_{D} \\
\delta p & =\delta f+w_{p} \\
\delta p r_{i} & =-\frac{1}{T_{s t}} \delta p r_{i}+w_{s r, i} \quad i=1,2,3,4 \\
\delta a_{D} & =-\frac{1}{T_{f}} \delta f+w_{f} \\
\delta a_{E} & =-\frac{1}{T_{a E}} \delta a_{E}+w_{a E} \\
\delta a_{N} & =-\frac{1}{T_{a N}} \delta a_{N}+w_{a N}+w_{a D} \\
\dot{T}_{a D} &
\end{aligned}
$$

where the error states $(x$, see eqn. (6)) are:

$\delta r_{N}, \delta r_{E}, \delta r_{D}-$ position errors north, east, down

$\delta v_{N}, \delta v_{E}, \delta v_{D}-$ velocity errors north, east, down

$\delta p, \delta f$-errors in UE clock phase and frequency errors

$\delta a_{N}, \delta a_{E}, \delta a_{D}$-acceleration error states north, east, down

$\delta h_{a}$--error in altitude measurement bias error

$\delta r_{i}$ - bias error in the pseudorange for $\operatorname{SV} i(i=1,2,3,4)$

and $w_{i}$ and $T_{i}$ are white noise sequences and correlation times respectively. 
The relationships between the receiver position errors in the local level system and the whole value position estimates $(\hat{L}, \hat{l}, \hat{r})$ in the GPS receiver are given below.

$$
\left.\begin{array}{l}
\delta r_{N}=(\hat{L}-L) r \\
\delta r_{E}=(\hat{l}-l) r \cos (L) \\
\delta r_{D}=-(\hat{r}-r)
\end{array}\right\}
$$

where

$$
\begin{aligned}
& L \text {-latitude } \\
& I \text {-longitude } \\
& r \text {-distance from earth centre. }
\end{aligned}
$$

Table 2 gives the initial standard deviation of the error states. The Table also presents the time constants and the white driving noise for the error states where it is applicable. The PSD of the white driving noise for the error states modeled as exponentially correlated noise, is derived from the linear variance equation of the system. To achieve a specified stationary error variance the PSD of the white driving noise is determined from the stationary solution of the variance equation. For a scalar case the relationship is (Gelb 1974):

$$
q=\frac{2 \sigma^{2}}{T}
$$

where

$q$-PSD of white driving noise

$\sigma^{2}$-the wanted variance of the stationary error state

$T$-correlation time

By initializing the time correlated error states according to eqn. (13), the error state will be in a stationary state from the start.

Additional comments to Table 2 are presented. The initial value of the position error down $(50 \mathrm{~m})$ represents the standard deviation of the difference between the geoide (sea level) and the WGS-72 reference ellipsoid which is utilized by GPS. The whole value altitude bias state $\left(h_{a}\right)$ represents this difference as the fact that the vessel is on the surface of the sea is used as altitude measurement.

The PSD of the white driving noise on the UE phase was selected to represent the corresponding error in the receiver clock. The initial value $(0.1 \mathrm{~m} / \mathrm{s})$ and the time constant $(5 \mathrm{~s})$ of the frequency error reflects the heavy wave induced error generated in the user clock due to the $g$-sensitivity of $10^{-9} / g$.

The initial values and the time constants of the acceleration states reflect the manoeuvres that the frigate might perform. These acceleration states are not expected to estimate wave induced accelerations to any large degree.

The pseudorange error bias states belong to the category of error states that would not be included in an actual receiver as explained in $\S 4.2$. In this case the biases are not observable, meaning that useful estimates are not generated. However, they do contribute to give Kalman filter covariances and Kalman gains closer to an 'optimal' system. 


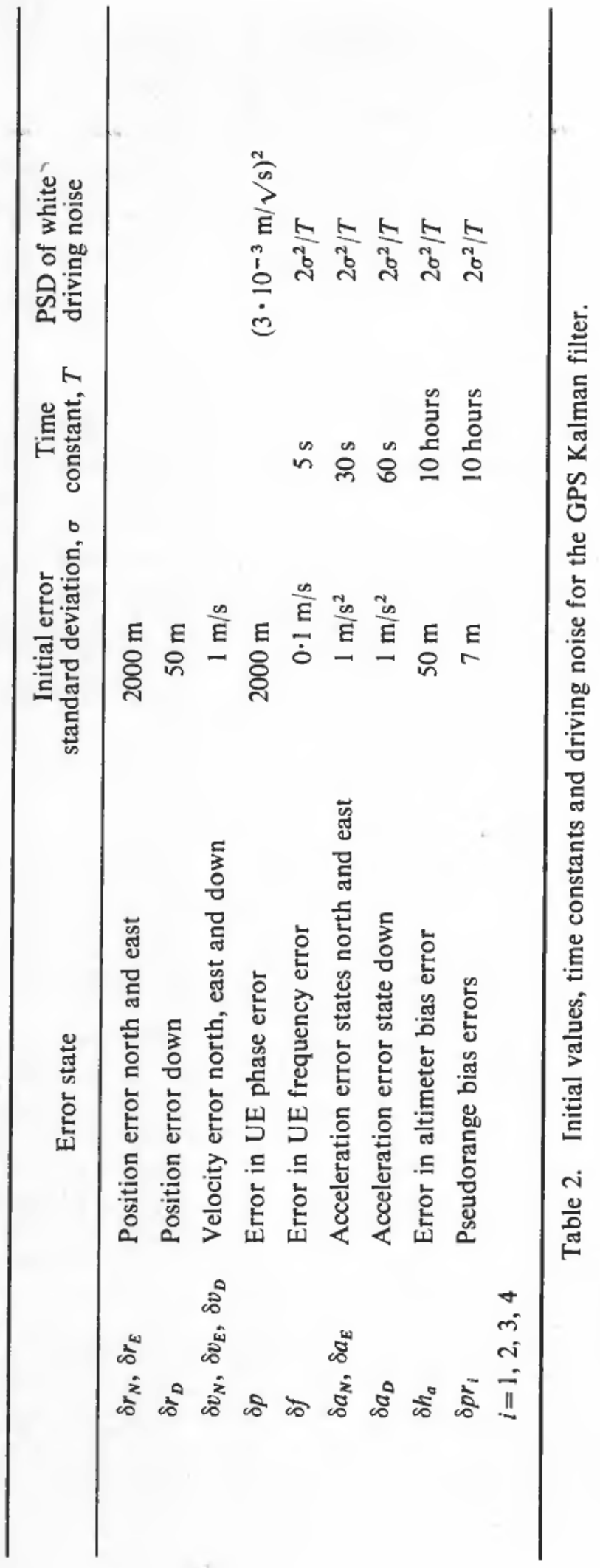




\subsection{Time propagation of GPS filter}

Between measurements the whole value navigation states are propagated using a set of dynamic equations. The whole value navigation states in the GPS receiver were chosen to be:

Latitude, longitude $(L, l)$

Distance from earth centre

(r)

Velocity north, east, down $\left(v_{N}, v_{E}, v_{D}\right)$

UE clock phase and frequency errors $(p, f)$

Acceleration north, east, down $\left(a_{N}, a_{E}, a_{D}\right)$

Bias in altitude measurement

$\left(h_{a}\right)$

The dynamic equations updating these variables are essentially the same as those given for the time update of the receiver Kalman filter. Figure 4 shows a diagram of the flow of data in the GPS simulation.

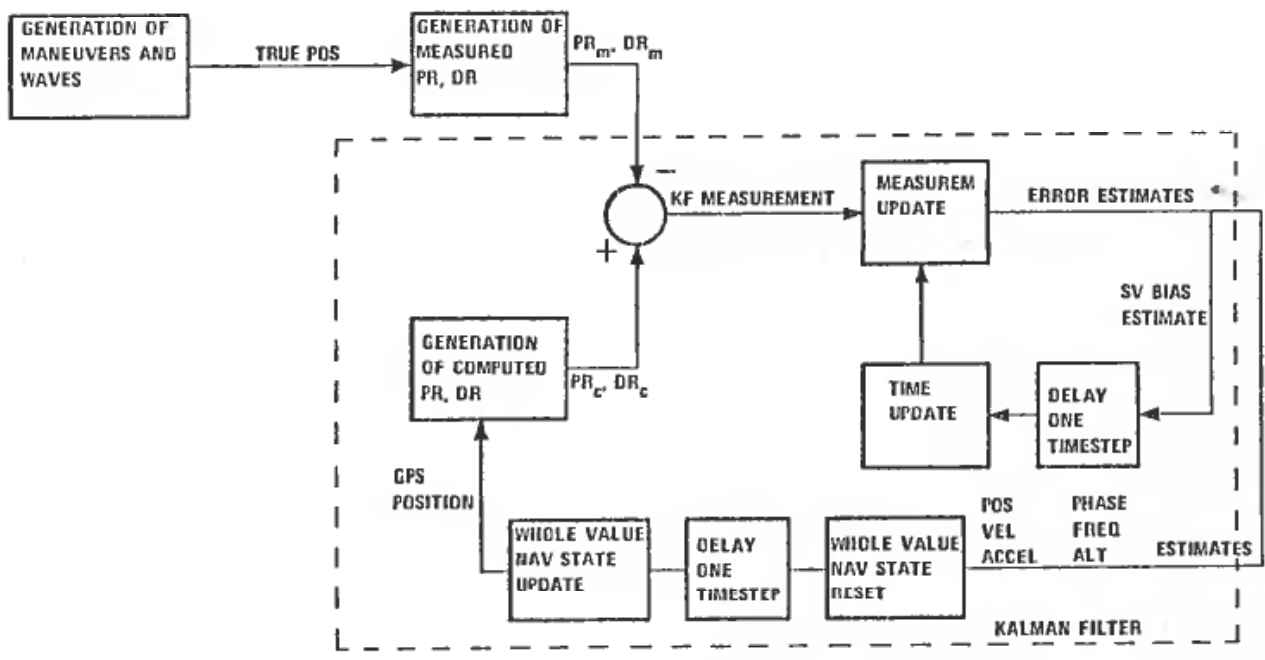

Figure 4. Data flow in the GPS simulation.

\subsection{GPS Kalman filter measurement model}

The measurement model of a Kalman filter may be derived by taking the partial derivative of the measurement to the Kalman filter with respect to the filter state.

The pseudorange measurement to the Kalman filter for satellite $i$ is defined as:

where

$$
\left.\begin{array}{l}
z_{P R, i}=P \hat{R}_{i}-P R_{i} \\
z_{P R, i}=\left(\hat{R}_{i}+\hat{p}\right)-\left(R_{i}+p+\delta p r_{i}+w_{i}\right)
\end{array}\right\}
$$

$P \hat{R}_{i}, P R_{i}-$ computed and measured pseudorange to SV $i$

$\hat{R}_{i}, R_{i}$-receiver computed and true range to SV $i$ from the receiver

$\hat{p}, p$-receiver computed and true UE clock phase error

$\delta p r_{i}$-bias in pseudorange measurement to SV $i$

$w_{i}$-white noise in pseudorange measurement 
By series expansion to first order of $\hat{R}_{i}$ around $R_{i}$ and using eqn. (12) and the definition $\delta p=\hat{p}-p$ one gets:

$$
z_{P R . i} \approx-u_{N i} \delta r_{N}-u_{E i} \delta r_{E}-u_{D i} \delta r_{D}+\delta p-\delta p r_{i}-w_{i}
$$

where $\left[u_{N i}, u_{E i}, u_{D i}\right]^{T}$ is the unit vector from the receiver to SV $i$.

The pseudorange measurement matrix is defined as:

$$
H_{P R, i}=\frac{\partial z_{P R, i}}{\partial x}
$$

where $\boldsymbol{x}$ is the Kalman filter error state vector on the left-hand side of eqn. (11). This yields for the pseudorange measurement matrices to the four SVs:

$$
\left.\begin{array}{l}
H_{P R, 1}=\left[-u_{N 1},-u_{E 1},-u_{D 1}, 0,0,0,1,0,0,0,0,0,-1,0,0,0\right] \\
H_{P R, 2}=\left[-u_{N 2},-u_{E 2},-u_{D 2}, 0,0,0,1,0,0,0,0,0,0,-1,0,0\right] \\
H_{P R, 3}=\left[-u_{N 3},-u_{E 3},-u_{D 3}, 0,0,0,1,0,0,0,0,0,0,0,-1,0\right] \\
H_{P R .4}=\left[-u_{N 4},-u_{E 4},-u_{D 4}, 0,0,0,1,0,0,0,0,0,0,0,0,-1\right]
\end{array}\right\}
$$

When the measurement update has been performed, the whole value navigation states are reset using the newly generated error states. It should be noted that there are no whole value navigation states for the error states $\delta p r_{i}$ in the error state vector. This means that the bias estimate is accumulated in the error estimate (which in this case is not an observable error). The significance of this is that e.g. in the UE phase case the Kalman filter is trying to estimate the error in the whole value navigation state, while in the SV bias case the Kalman filter is trying to estimate the entire bias. This is illustrated in Fig. 4 where the SV bias and the other states are updated in different feedback loops. This is also why the pseudorange measurement matrix elements corresponding to the UE phase error and the SV bias have different signs.

The deltarange measurement matrix was derived using a technique wherc the linear perturbation relation between deltarange and the filter state vector $\boldsymbol{x}$ was used:

$$
d z_{D R, i}=H_{P R, i}\left(t_{2}\right) d x\left(t_{2}\right)-H_{P R, i}\left(t_{1}\right) d x\left(t_{1}\right)
$$

$d z_{D R, i}$-differential of the deltarange measurement to SV $i$

$d x\left(t_{1}\right), d x\left(t_{2}\right)$ - differential of the error state at deltarange start $\left(t_{1}\right)$ and deltarange stop $\left(t_{2}\right)$

$H_{P R . i}\left(t_{1}\right), H_{P R . i}\left(t_{2}\right)$-pseudorange measurement matrix at deltarange start and stop

The deltarange measurement matrix referenced to deltarange stop will now be:

But now

$$
H_{D R . t}=\frac{\partial z_{D R, i}}{\partial \boldsymbol{x}\left(t_{2}\right)}=H_{P R, i}\left(t_{2}\right)-H_{P R . i}\left(t_{1}\right) \frac{\partial x\left(t_{1}\right)}{\partial x\left(t_{2}\right)}
$$

$$
\left.\begin{array}{c}
\frac{\partial x\left(t_{1}\right)}{\partial x\left(t_{2}\right)}=\Phi\left(t_{1}-t_{2}\right)=\Phi(-\Delta t) \\
\Delta t=t_{2}-t_{1}
\end{array}\right\}
$$


$\Phi(\Delta t)$ is the transition matrix (see eqn. (7)) which is approximated using:

$$
\Phi(\Delta t) \approx I+F \Delta t+\frac{1}{2} F^{2} \Delta t^{2}
$$

Assuming that the deltarange interval $\Delta t$ is much shorter than the shortest time constants in the error state dynamics ( $F$-matrix, see eqn. (6)), one gets for the relevant terms in the transition matrix:

$$
\left.\begin{array}{l}
\Phi_{1,1}(\Delta t)=\Phi_{2,2}(\Delta t)=\Phi_{3,3}(\Delta t)=\Phi_{7,7}(\Delta t) \approx 1 \\
\Phi_{1,4}(\Delta t)=\Phi_{2,5}(\Delta t)=\Phi_{3,6}(\Delta t)=\Phi_{7,8}(\Delta t) \approx \Delta t \\
\Phi_{1,9}(\Delta t)=\Phi_{2,10}(\Delta t)=\Phi_{3,11}(\Delta t) \approx \frac{1}{2} \Delta t^{2}
\end{array}\right\}
$$

Inserting eqns. (19) and (20) in eqn. (18) yields:

$$
\begin{array}{r}
H_{D R . i}=\left[-\Delta u_{N i},-\Delta u_{E i},-\Delta u_{D i},-\Delta t u_{N i}\left(t_{1}\right),-\Delta t u_{E i}\left(t_{1}\right),-\Delta t u_{D i}\left(t_{1}\right),\right. \\
\left.0, \Delta t, \frac{1}{2} \Delta t^{2} u_{N i}\left(t_{1}\right), \frac{1}{2} \Delta t^{2} u_{E i}\left(t_{1}\right), \frac{1}{2} \Delta t^{2} u_{D i}\left(t_{1}\right), 0,0,0,0,0\right]
\end{array}
$$

where

$$
\left.\begin{array}{l}
\Delta u_{N i}=u_{N i}\left(t_{2}\right)-u_{N i}\left(t_{1}\right) \\
\Delta u_{E i}=u_{E i}\left(t_{2}\right)-u_{E i}\left(t_{1}\right) \\
\Delta u_{D i}=u_{D i}\left(t_{2}\right)-u_{D i}\left(t_{1}\right)
\end{array}\right\}
$$

The measurement matrix for the altitude measurement will be derived below.

The altitude measurement to the GPS Kalman filter is:

$$
\left.\begin{array}{l}
z_{a}=\hat{h}-h \\
z_{a}=\left(\hat{r}+\hat{h}_{a}\right)-\left(r+h_{a}+w_{a}\right) \\
z_{a}=-\delta r_{D}+\delta h_{a}-w_{a}
\end{array}\right\}
$$

where

$\hat{h}, h$-computed and measured altitude

$\hat{r}, r$-computed and true distance from earth centre

$\hat{h}_{a}, h_{a}-$ computed and true bias in altitude measurement

$w_{a}$-white noise on altitude measurement

$\delta r_{D}-$ position error down

$\delta h_{a}-$ error in altitude measurement bias error

The altitude measurement matrix is now:

$$
\left.\begin{array}{l}
H_{a}=\frac{\partial z_{a}}{\partial \boldsymbol{x}} \\
H_{a}=[0,0,-1,0,0,0,0,0,0,0,0,1,0,0,0,0,]
\end{array}\right\}
$$

The standard deviation of the random measurement noise in the filter is given in Table 3. 


\begin{tabular}{ll}
\hline Pseudorange measurement & $2 \mathrm{~m}$ \\
Deltarange measurement & $0.02 \mathrm{~m}$ \\
Altitude measurement & $5 \mathrm{~m}$ \\
\hline
\end{tabular}

Table 3. Standard deviation of GPS Kalman filter measurement noise.

\section{Selected simulation results}

This section contains some selected simulation results showing a parallel and a sequential GPS receiver operating alone and integrated with an attitude reference system. Also included is a simulation of navigation during satellite outage caused by mountain shadowing. The simulations presented were performed using a reference scenario as a baseline. This scenario is defined below:

$\begin{array}{ll}\text { Type of vessel } & \text { frigate } \\ \text { Vessel speed } & 20 \text { knots } \\ \text { Vessel heading } & 0^{\circ} \text { (due north) } \\ \text { Significant wave height } & 4 \mathrm{~m} \\ \text { Direction of wave encounter } & 105^{\circ} \text { (nearly on the beam) }\end{array}$

The parallel receiver simulated gets a measurement from each SV every second while the sequential receiver sequences through the four SVs in four seconds.

\subsection{Unaided GPS performance}

Figure 5 shows the position and velocity errors (Monte Carlo standard deviations calculated from 100 single runs) in an unaided GPS receiver in the scenario described above. It is noted that the velocity accuracy is very poor, being slightly better for a parallel receiver than for a sequential. However, the accuracy in both cases is too poor to be useful. The reason for the better accuracy in north velocity for the parallel receiver is that the vessel is going north with the waves encountered almost on the beam. This results in the uncompensated roll movements to affect the east-west direction more heavily than the north-south direction.

\subsection{Lever arm compensation}

Several simulations were performed were the vessel attitude was provided to the GPS receiver by an attitude reference system to compensate for the rotational movements of the vessel. Figure 6 shows the position and velocity error north in this case. The plots show single time simulation runs (contrary to Monte Carlo standard deviations in the previous figure).

The dramatic difference in the velocity error of the parallel and sequential receiver is noted. (The velocity error is typically $0.1 \mathrm{~m} / \mathrm{s}$ using the parallel receiver.) The reason for this is that the sequential receiver is not able to track the linear wave induced motions (surge, sway, heave). These motions have a typical period of 5 seconds in the simulated scenario while the sequential receiver gets a complete new update only every 4 seconds.

The parallel receiver on the other hand gets a complete new update every one second and is therefore able to track these linear motions. 

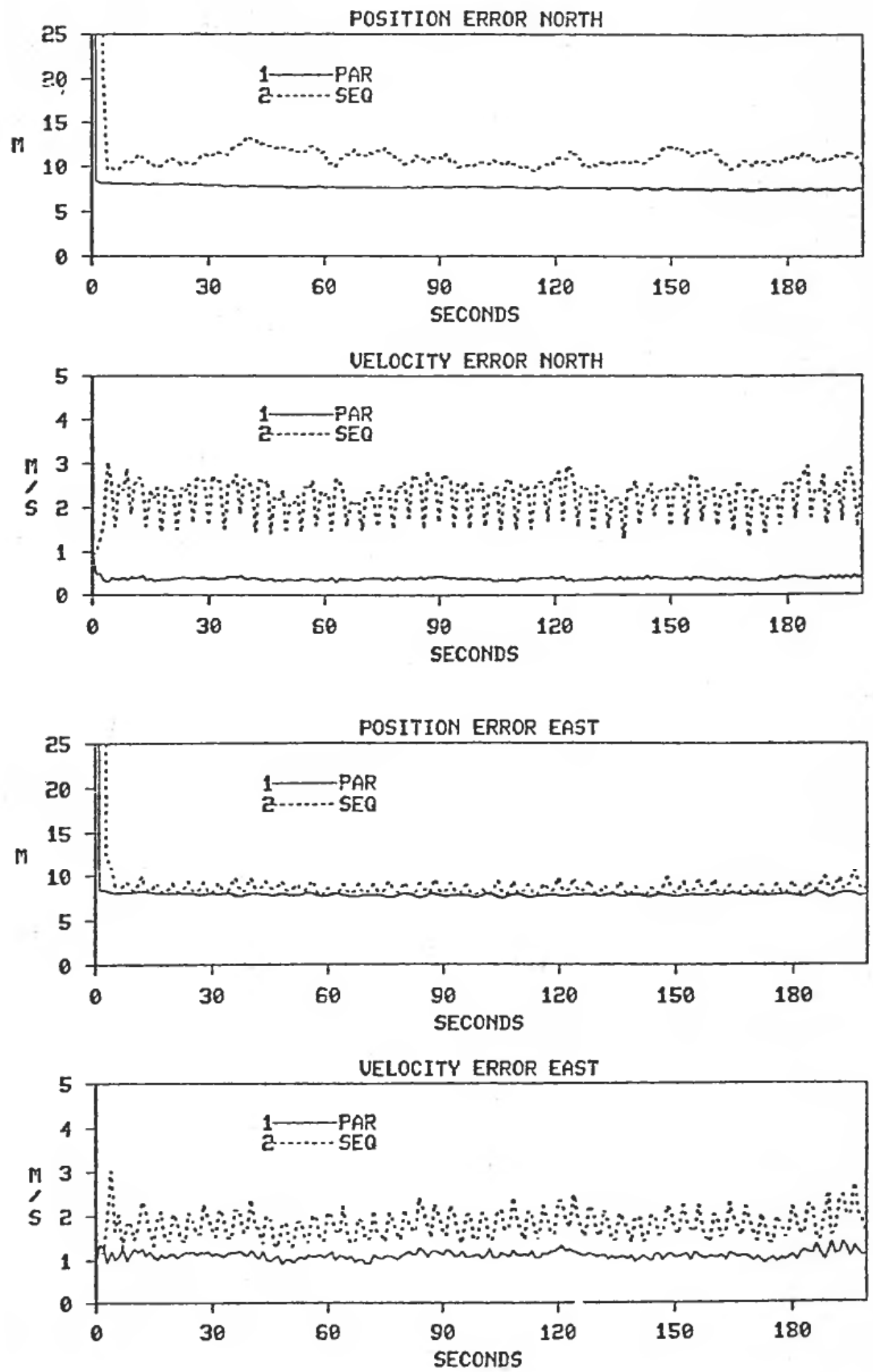

Figure 5. Standard deviation of position and velocity errors for GPS on its own. 

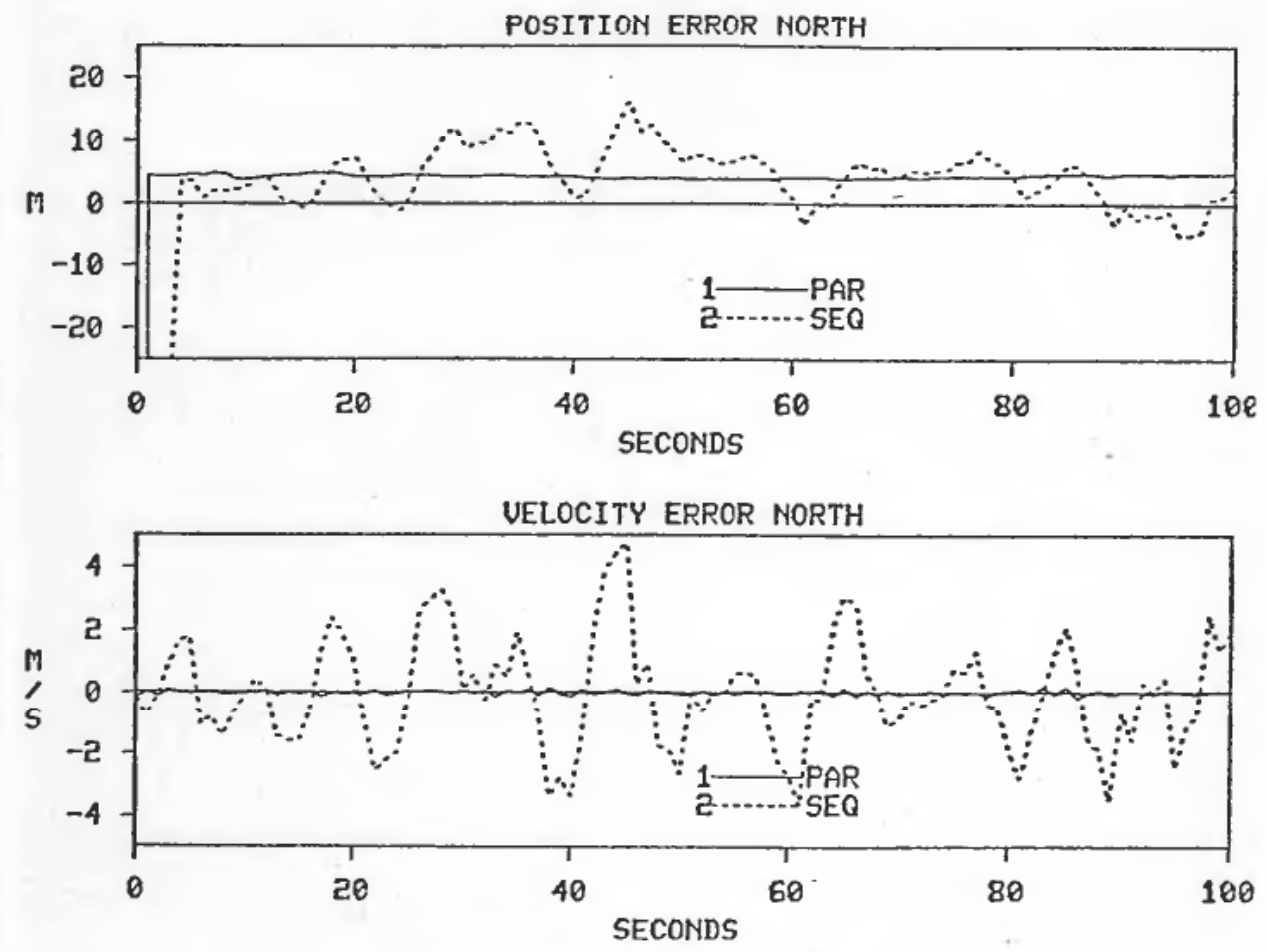

Figure 6. Single time simulation when using GPS and only lever arm compensation.

Different accuracy levels of the attitude information provided to the GPS receiver were also simulated. It turned out that the attitude accuracy was not very critical. An attitude accuracy of $2 \mathrm{~min}$ of arc and $\mathrm{I}$ degree provided almost the same position and velocity accuracy.

\subsection{Navigating in fjords}

A look at the map of Norway reveals that when navigating in a fjord, masking angles of $20^{\circ}$ or more may be experienced (i.e. no SVs below $20^{\circ}$ elevation are seen).

When navigating on the ocean surface only $3 \mathrm{SVs}$ are necessary as the altitude is known and only horizontal position is of interest. Figure 7 shows the HDOP (see Appendix A) and number of visible satellites for different masking angles at $60^{\circ} \mathrm{N}$ and $5^{\circ} \mathrm{E}$. (Epoch is a random reference time.) It is noted that only $2 \mathrm{SVs}$ are visible at times using $20^{\circ}$ mask angle.

Figure 8 shows the expected position error north with the $20^{\circ}$ mask angle, starting 100 minutes past epoch and assuming a ranging error of $7 \mathrm{~m}$. Figure 9 shows the corresponding actual position error using a GPS receiver on its own. The plus and minus Kalman filter indicated standard deviations together with a single time run is shown using a sequential receiver. Only range measurements are used as the velocity accuracy is too poor to be useful because of wave induced motions.

Comparing the Kalman filter standard deviation in Fig. 9 to the expected error calculated from HDOP in Fig. 8 it is seen that there is not a very good agreement. According to Fig. 8 the position solution should become very poor at the end of 


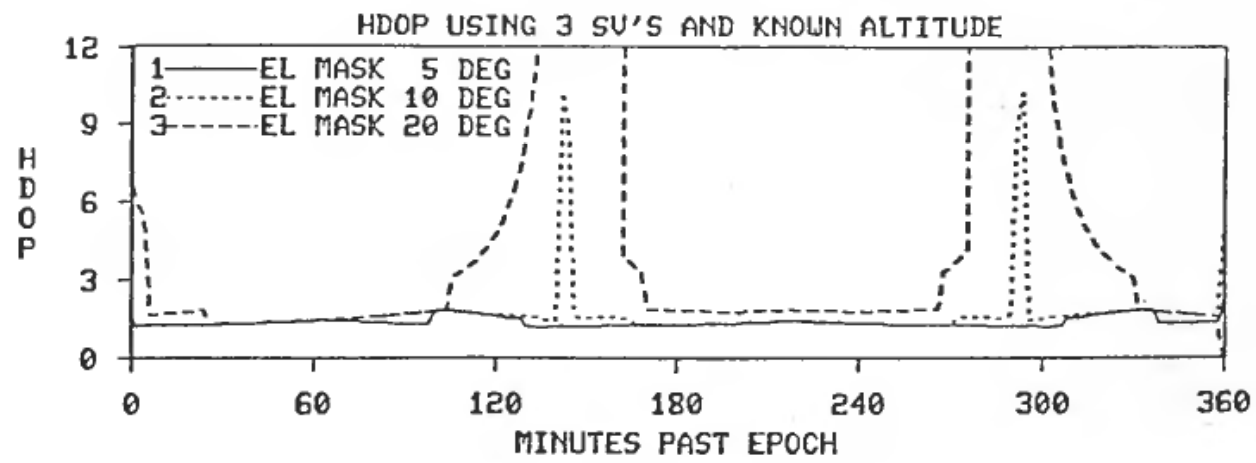

NO OF SU'S UISIBLE

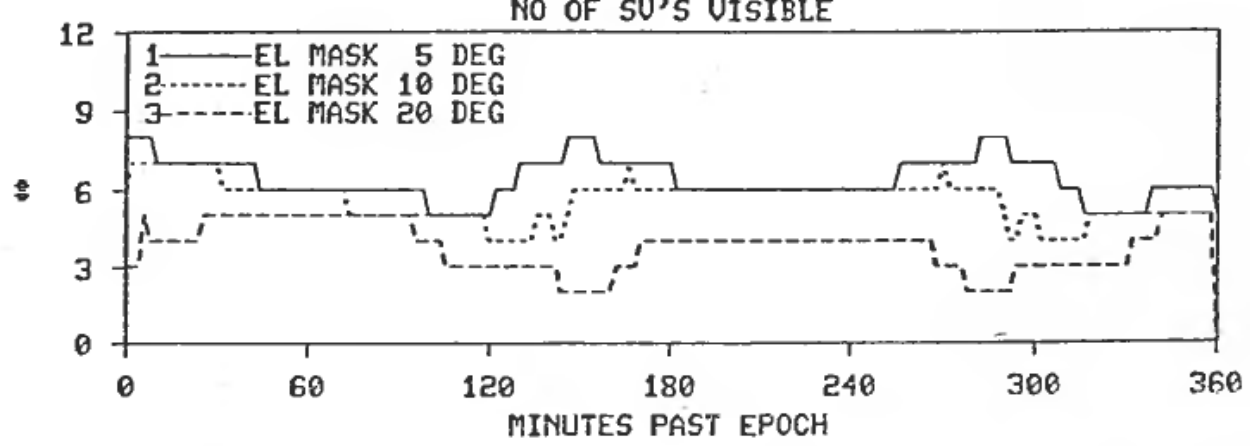

Figure 7. HDOP using three SVs and known altitude for different elevation mask angles at $60^{\circ} \mathrm{N} 5^{\circ} \mathrm{E}$. Also shown is the total number of SVs visible.

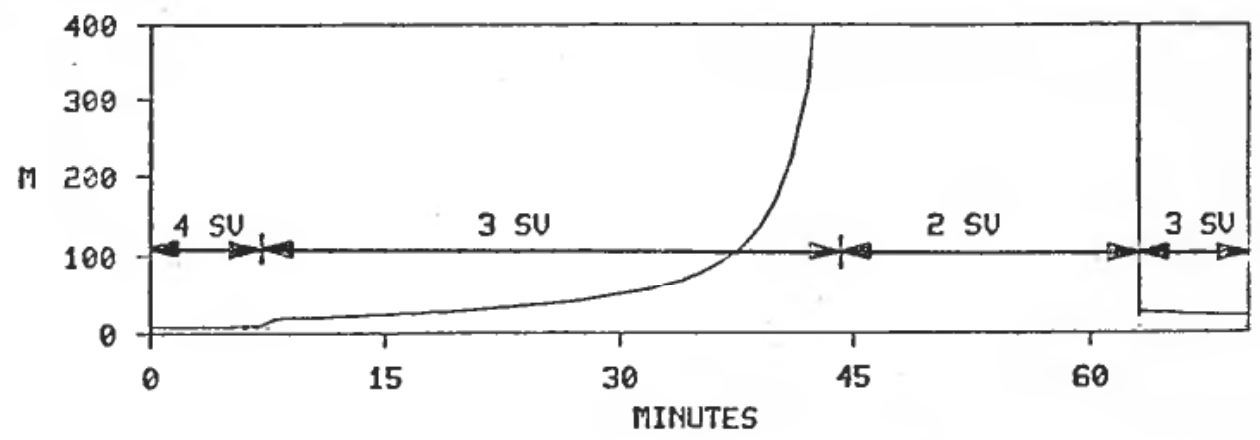

Figure 8. Expected standard deviation of position error north calculated from HDOP using three SVs and known altitude. The numbers of visible SVs are also indicated.

the $3 \mathrm{SV}$ period and no HDOP is calculated during the $2 \mathrm{SV}$ period as there is not enough information available for a solution.

The reason that the errors in Fig. 9 are fairly well behaved is that the user clock stability provides additional information. With a 100 times less accurate user clock, the result was in close agreement with what was expected from the plot in Fig. 8. At the other extreme, one would expect to be able to navigate well using a perfect clock and altitude information even using only 2 SVs with favourable geometry.

Considering the above the expected user position error calculated from HDOP is a conservative measure as it does not take into account that any GPS receiver has a 


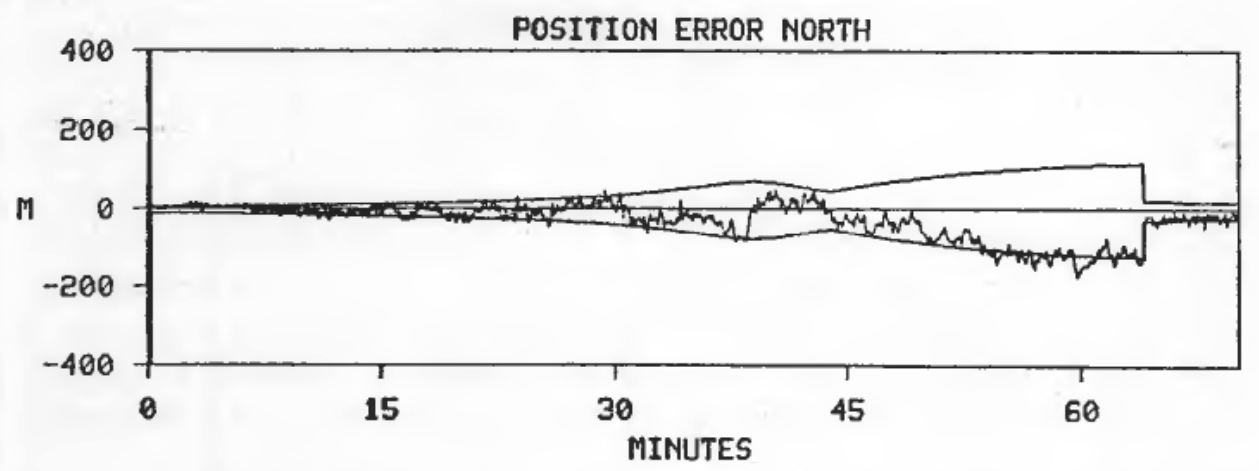

Figure 9. Time run simulation and plus/minus Kalman filter indicated standard deviation of position error north using GPS on its own during satellite outages. Altitude meaurement is used.

fairly stable oscillator. It may be noted that the oscillator was modeled with a frequency stability of $3 \cdot 10^{-10}(1 \sigma)$ in the Kalman filter. This was due to the heavy influence of the $g$-sensitivity in the simulated scenario.

\section{Conclusions}

The position errors of an unaided GPS receiver are fairly good $(\approx 10 \mathrm{~m} 1 \sigma)$, although somewhat more noisy with a sequential than a parallel receiver. The velocity errors are very bad $(>I \mathrm{~m} / \mathrm{s} l \sigma)$ due to wave induced motions, again worse with a sequential receiver. Scenario conditions like type of vessel, speed and wave height $(4 \mathrm{~m}$ or $8 \mathrm{~m})$ do not have a great influence on accuracy.

Lever arm compensation does not improve the velocity accuracy of a sequential GPS receiver much as the receiver is not able to track the linear wave induced motions. However, lever arm compensation improves the velocity accuracy of a parallel receiver considerably (from $\approx 1 \mathrm{~m} / \mathrm{s}$ to $\approx 0.1 \mathrm{~m} / \mathrm{s}$ ). The attitude accuracy used in the lever arm correction is not critical. Navigating in fjords with a GPS receiver on its own with reduced number of SVs visible provides better accuracy than would be expected from HDOP calculations. This is due to the additional information provided by the fairly stable oscillator in the receiver.

\section{Appendix A}

\section{GDOP calculations}

This appendix presents a short description of the geometric dilution of precision (GDOP) parameters as a measure of the effect of satellite geometry on GPS navigation fix errors.

In $\S 4.5$ the pseudorange measurement matrix to four SVs may be found. By omitting all other errors except the three position error components and the clock phase bias error one may write:

$$
G x=z \text { or } \boldsymbol{x}=G^{-1} z
$$


where

$$
G=\left[\begin{array}{llll}
-u_{N 1} & -u_{E 1} & -u_{D 1} & 1 \\
-u_{N 2} & -u_{E 2} & -u_{D 2} & 1 \\
-u_{N 3} & -u_{E 3} & -u_{D 3} & 1 \\
-u_{N 4} & -u_{E 4} & -u_{D 4} & 1
\end{array}\right]
$$

$\left[u_{N i}, u_{E i}, u_{D i}\right]^{T}$ - the unit vector from receiver to SV $i$

$$
z=\left[z_{P R, 1}, z_{P R, 2}, z_{P R, 3}, z_{P R, 4}\right]^{T}
$$

$z_{P R, i}-$ difference between computed and true pseudorange measurement for SV $i$

$$
x=\left[\delta r_{N}, \delta r_{E}, \delta r_{D}, \delta p\right]^{T}
$$

$\delta r_{N}, \delta r_{E}, \delta r_{D}-$ position error north, east and down

$\delta p-$ error in clock phase error

Equation (A 1) expresses the relationship between incremental pseudorange measurements and corrections that the user will make to his current estimate of position and clock phase bias. The incremental pseudorange measurements are the differences between the measurements that have been predicted by the user (based on knowledge of satellite position and the user's most current estimate of his position and clock bias) and the actual measured pseudoranges. As the relationship in eqn. (A I) is linear, it can be used to express the relationship between errors in pseudorange measurements and the user quantities:

$$
\delta x=G^{-1} \delta z
$$

where $\delta \boldsymbol{z}$ represents the pseudorange measurement errors and $\delta \boldsymbol{x}$ the corresponding errors in user position and clock bias. The covariance of the user position error is now:

$$
\left.\begin{array}{l}
\operatorname{cov}(\delta x)=E\left\{\delta x \delta x^{T}\right\}=E\left\{\left(G^{-1} \delta z\right)\left(G^{-1} \delta z\right)^{T}\right\} \\
\operatorname{cov}(\delta x)=G^{-1} E\left\{\delta z \delta z^{T}\right\} G^{-T} \\
\operatorname{cov}(\delta x)=G^{-1} \operatorname{cov}(\delta z) G^{-T}
\end{array}\right\}
$$

where

$$
\begin{array}{ll}
\operatorname{cov}(\delta x)=E\left\{\delta \boldsymbol{x} \delta \boldsymbol{x}^{T}\right\} & \text { user error covariance } \\
\operatorname{cov}(\delta z)=E\left\{\delta z \delta z^{T}\right\} & \text { pseudorange error covariance }
\end{array}
$$

The symbol $E\{\}$ designates 'expected value'.

The matrix $G$ is determined by the satellite geometry. From eqn. (A 3 ) it is seen that the relationship between pseudorange measurement errors and the user's position and clock bias errors is a function only of the matrix $G$. That is, the error relationship is a function only of satellite geometry. $\operatorname{cov}(\delta z)$ reflects range measurement error statistics, e.g. satellite ephemeris errors, propagation errors and receiver errors. In order to arrive at a measure containing only the effects of geometry, $\operatorname{cov}(\delta z)$ in eqn. (A 3 ) is assumed equal to the identity matrix. This means that each individual pseudorange measurement has a $1 \sigma$ error of unity, where the expected mean 
is zero and the correlations of errors between satellites are also zero. Thus, for this case, the covariance matrix for user position and clock bias errors is given by:

$$
\operatorname{cov}(\delta x)=G^{-1} G^{-T}=\left(G^{T} G\right)^{-1}
$$

GDOP is defined as:

$$
\text { GDOP }=\left(\text { TRACE }\left[\left(G^{T} G\right)^{-1}\right]\right)^{1 / 2}
$$

The diagonal elements of $\operatorname{cov}(\delta x)$ are the variances of estimated user position error in each axis and in the user time offset. The GDOP may then be defined as:

$$
\mathrm{GDOP}=\left(\sigma_{x}{ }^{2}+\sigma_{y}{ }^{2}+\sigma_{z}{ }^{2}+\sigma_{t}{ }^{2}\right)^{1 / 2}
$$

where

$$
\begin{gathered}
\sigma_{x}^{2}, \sigma_{y}^{2}, \sigma_{z}^{2} \text {-variances of estimated user position assuming unity range error } \\
\sigma_{t}{ }^{2}-\text { variance of estimated time offset assuming unity range error }
\end{gathered}
$$

GDOP is, in effect, the multiplication factor of pseudorange measurement errors into user errors due to the effect of satellite geometry. GDOP may also be used to select the four best satellites from those that are visible.

For navigation purposes knowing the exact time is generally of secondary interest. Therefore the most frequently used criterion for satellite selection is the position dilution of precision (PDOP) defined as:

$$
\mathrm{PDOP}=\left(\sigma_{x}{ }^{2}+\sigma_{y}{ }^{2}+\sigma_{z}{ }^{2}\right)^{1 / 2}
$$

PDOP multiplied by the user to satellite range error gives the radial error in user position in three dimensions. Similarly one may define:

$$
\begin{array}{ll}
\text { HDOP } & =\left(\sigma_{x}{ }^{2}+\sigma_{y}{ }^{2}\right)^{1 / 2} \text { - } \text { horizontal dilution of precision } \\
\text { VDOP }=\sigma_{z} & \text {-vertical dilution of precision } \\
\text { TDOP }=\sigma_{t} & \text { - time dilution of precision }
\end{array}
$$

$\begin{array}{ll}\text { Abbreviations } & \\ \text { CEP } & \text { Circular Error Probable (50\%) } \\ \text { C/A } & \text { Clear or Coarse/Acquisition } \\ \text { DR } & \text { Deltarange } \\ \text { E } & \text { East } \\ \text { GPS } & \text { Global Positioning System } \\ \text { HDOP } & \text { Horizontal Dilution of Precision } \\ \text { ITTC } & \text { International Towing Tank Conference } \\ \text { JPO } & \text { Joint Program Office } \\ \text { MOU } & \text { Memorandum of Understanding } \\ \text { N } & \text { North } \\ \text { NATO } & \text { North Atlantic Treaty Organization } \\ \text { P } & \text { Protected or Precision } \\ \text { PDOP } & \text { Position Dilution of Precision } \\ \text { PR } & \text { Pseudorange } \\ \text { PSD } & \text { Power Spectral Density } \\ \text { RMS } & \text { Root Mean Square } \\ \text { SV } & \text { Space Vehicle }\end{array}$


TDOP Time Dilution of Precision

UE User Equipment

US United States

WGS-72 World Geodetic System of 1972

\section{REFERENCES}

BARDAL, J., and ØRPEN, O. Ø. (1982). Integration of NAVSTAR GPS and Inertial Navigation for Marine Applications. FFI/RAPPORT-82/7002. Norwegian Defence Research Establishment.

BiermanN, G. J. (1977). Factorization Methods for Discrete Sequential Estimation (New York: Academic Press).

GelB, A. (1974). Applied Optimal Estimation (Cambridge, Massachussets: The MIT Press).

PrICE, W. G., et al. (1974). Probabilistic Theory of Ship Dynamics (London: Chapman and Hall). 